\title{
INTEGRALLY CLOSED AND COMPONENTWISE LINEAR IDEALS
}

\author{
A. CONCA, E. DE NEGRI, M. E. ROSSI
}

\begin{abstract}
In a two dimensional regular local ring integrally closed ideals have a unique factorization property and their associated graded ring is CohenMacaulay. In higher dimension these properties do not hold and the goal of the paper is to identify a subclass of integrally closed ideals for which they do. We restrict our attention to 0 -dimensional homogeneous ideals in polynomial rings $R$ of arbitrary dimension. We identify a class of integrally closed ideals, the Goto-class $\mathcal{G}^{*}$, which is closed under product and it has a suitable unique factorization property. Ideals in $\mathcal{G}^{*}$ have a Cohen-Macaulay associated graded ring if either they are monomial or $\operatorname{dim} R \leq 3$. Our approach is based on the study of the relationship between the notions of integrally closed, contracted, full and componentwise linear ideals.
\end{abstract}

\section{INTRODUCTION}

Thanks to the work of Zariski, integrally closed ideals of two-dimensional regular local rings $(R, \mathbf{m})$ are well-understood. In such rings the product of integrally closed ideals is integrally closed and there is a unique factorization property for integrally closed ideals into product of simple integrally closed ideals. In higher dimension, these properties no longer hold, see the examples in $[\mathrm{C}, \mathrm{C} 3, \mathrm{H}, \mathrm{L}]$. The identification of analogues of Zariski's results is an active research area. In this direction we mention the work of Cutkosky $[\mathrm{C}, \mathrm{C1}, \mathrm{C2}, \mathrm{C} 3$, Deligne $[\mathrm{D}$, Huneke $\mathrm{H}$ ] and Lipman $[\mathrm{L}$. Several authors considered other related problems, as for instance the description of integrally closed ideals $I$ such that $I \mathbf{m}$ is integrally closed as well, see CGPU, DC1, DC2, EM, Ga, Ga1, HH].

In this paper we deal with homogeneous ideals of $R=K\left[x_{1}, \ldots, x_{n}\right]$, the polynomial ring over a field $K$. For an ideal $I$ we denote by $o(I)$ the order or initial degree of $I$, by $I_{j}$ the homogeneous component of degree $j$ of $I$ and by $I_{\langle j\rangle}$ the ideal generated by $I_{j}$. We set $\mathbf{m}=\left(x_{1}, \ldots, x_{n}\right)$.

Our goal is to identify a class of $\mathbf{m}$-primary integrally closed ideals of $R$ which behaves, as much as possible, as the class of integrally closed ideals in dimension 2. To this end, we study the relations between four properties of ideals: 1) being integrally closed, 2) being componentwise linear, 3) being contracted (from a quadratic extension), 4) being $\mathbf{m}$-full. It turns out that 1) implies 3 ), that 2) implies 3 ) and that 3) implies 4). Also, for ideals $I$ such that $I+(\ell)=\mathbf{m}^{o(I)}+(\ell)$ for some linear form $\ell$, one has that 4) implies 2).

2000 Mathematics Subject Classification. Primary 13B22; Secondary 13D02.

Key words and phrases. integrally closed ideals, associated graded rings, componentwise linear ideals, factorization properties.

Research partially supported by the "Ministero dell'Universitá e della Ricerca Scientifica" in the framework of the National Research Network (PRIN 2005) "Algebra commutativa, combinatorica e computazionale". 
We then consider the class $\mathcal{C}$ of the $\mathbf{m}$-primary ideals of $R$ satisfying $I+(\ell)=$ $\mathbf{m}^{o(I)}+(\ell)$ for some linear form $\ell$ and having property 4 ), (equivalently 3 ) or 2$)$ ). Denote by $\mathcal{C}^{*}$ the set of the ideals in $\mathcal{C}$ that are integrally closed. We prove that $\mathcal{C}$ is closed under product and integral closure, see Proposition 3.5. Further, we prove in Theorem 3.13 that $\mathcal{C}$ has a factorization property that looks like Zariski's factorization for contracted ideals in dimension 2 [ZS, Appendix 5, Thm.1]. An important role in Zariski's factorization theorem is played by the characteristic form $g(I)$ defined has the GCD of the forms of degree $o(I)$ in $I$. Given $I \in \mathcal{C}$ for every $j \in \mathbf{N}$ we define $Q_{j}(I)$ to be the saturation of $I_{\langle j+o(I)\rangle}$. In our context, the characteristic form is replaced by the ideal $Q_{0}(I)$.

We show that given $I \in \mathcal{C}$, one has $I \in \mathcal{C}^{*}$ iff $I \mathbf{m} \in \mathcal{C}^{*}$. But, unfortunately, $\mathcal{C}^{*}$ is not closed under product. We then consider the Goto-class $\mathcal{G}$ defined as the set of the ideals $I \in \mathcal{C}$ such that for every $j$ the primary components of $Q_{j}(I)$ are powers of (necessarily 1-dimensional) geometrically prime ideals. Integrally closed complete intersections, characterized by Goto $\mathrm{G}$, are in $\mathcal{G}$, see Theorem [4.9. We prove in Proposition 4.7 that $\mathcal{G}$ is closed under product and that it is compatible with the factorization of $\mathcal{C}$. We define $\mathcal{G}^{*}$ to be the set of the integrally closed ideals of $\mathcal{G}$. We then show that $\mathcal{G}^{*}$ is closed under product and has a unique factorization property, see Theorem 4.8. The simple elements in $\mathcal{G}^{*}$ have a "simple" description: up to a change of coordinates, they are of the form $\overline{\left(x_{1}^{d}, \ldots, x_{n-1}^{d}, x_{n}^{t}\right)}$ for coprime $d, t$ with $d<t$. Lipman and Teissier [LT and Huneke H2 proved that integrally closed ideals in two dimensional regular local rings have a Cohen-Macaulay associated graded ring. It is natural to ask whether the same holds for ideals of $\mathcal{G}^{*}$. We conclude the paper by showing that if $I \in \mathcal{G}^{*}$ and either $I$ is monomial (e.g. $Q_{0}(I)$ has at most two minimal primes) or $\operatorname{dim} R \leq 3$, then the associated graded ring $\operatorname{gr}_{I}(R)$ is Cohen-Macaulay, see Corollary 4.12 and Theorem 4.14 .

\section{2. $\mathbf{m}$-FULL, CONTRACTED AND COMPONENTWISE LINEAR IDEALS}

Throughout the paper let $R=K\left[x_{1}, \ldots, x_{n}\right]$ be a polynomial ring over a field $K$, and $\mathbf{m}=\left(x_{1}, \ldots, x_{n}\right)$. All the ideals we deal with are homogeneous (with few exceptions).

Let $I$ be an ideal of $R$. Denote by $\mu(I)$ the minimum number of generators of $I$ and by $o(I)$ the initial degree (or the order) of $I$, that is the least degree of non-zero elements in $I$.

In this section we discuss the relations between $\mathbf{m}$-full, contracted and componentwise linear ideals. First we introduce some notation and recall definitions. Denote by $\beta_{i j}(I)$ the $i j$-th graded Betti number of $I$ as an $R$-module. The CastelnuovoMumford regularity of $I$ is given by

$$
\operatorname{reg}(I)=\max \left\{j-i: \beta_{i j}(I) \neq 0\right\} .
$$

The ideal $I$ has a linear resolution if $\operatorname{reg}(I)=o(I)$. For general facts on the Castelnuovo-Mumford regularity and its characterization in terms of local cohomology we refer the reader to $\left[\mathrm{E}\right.$. For every integer $j$ denote by $I_{j}$ the $K$-vector space of the forms of degree $j$ in $I$, and by $I_{\langle j\rangle}$ the ideal generated by the elements of $I_{j}$. The ideal $I_{\langle j\rangle}$ has a linear resolution for $j \geq \operatorname{reg}(I)$.

Given two ideals $I$ and $J$, we set $I: J^{\infty}=\cup_{k} I: J^{k}$. We denote by $I^{\text {sat }}$ the saturation of $I$ with respect to $\mathbf{m}$, that is

$$
I^{\mathrm{sat}}=I: \mathbf{m}^{\infty} .
$$


For short we will denote the ideal $\left(I_{\langle j\rangle}\right)^{\text {sat }}$ by $I_{\langle j\rangle}^{\text {sat }}$.

Definition 2.1. An ideal $I \subset R$ is said to be componentwise linear if $I_{\langle d\rangle}$ has a linear resolution for every $d \in \mathbf{N}$.

For every non-zero linear form $\ell$ in $R$ we consider the quadratic transform $S$ of $R$ associated to $\ell$. By definition $S=R[\mathbf{m} / \ell]=\cup_{k \in \mathbf{N}} \mathbf{m}^{k} / \ell^{k}$.

Definition 2.2. An ideal $I \subset R$ is said to be contracted (from a quadratic extension) if there exists a non-zero linear form $\ell$ in $R$ such that $I=I S \cap R$, where $S=R[\mathbf{m} / \ell]$.

Proposition 2.3. Let $\ell$ be a non-zero linear form in $R$ and $I \subset R$ an ideal. Set $S=R[\mathbf{m} / \ell]$ and $J=I S \cap R$. We have:

(1) $J=\cup_{k \in \mathbf{N}}\left(I \mathbf{m}^{k}: \ell^{k}\right)$.

(2) $J$ is homogeneous.

(3) $J_{j}=\left(I_{\langle j\rangle}^{\mathrm{sat}}: \ell^{\infty}\right)_{j}$.

Proof. (1) follows immediately from the fact that $I S=\cup_{k} I \mathbf{m}^{k} / \ell^{k}$. Then (2) follows from (1). To prove (3) consider $f \in R$ homogeneous of degree $j$. We have $f \in J_{j}$ iff $f \ell^{k} \in\left(I \mathbf{m}^{k}\right)_{j+k}$ for every $k \gg 0$. Since $\left(I \mathbf{m}^{k}\right)_{j+k}=\left(I_{\langle j\rangle}\right)_{j+k}$ we have $f \in J_{j}$ iff $f \ell^{k} \in I_{\langle j\rangle}$ for every $k \gg 0$. Hence $f \in J_{j}$ iff $f \in I_{\langle j\rangle}: \ell^{\infty}=I_{\langle j\rangle}^{\text {sat }}: \ell^{\infty}$.

In the following we denote by $\operatorname{Ass}(M)$ the set of the associated prime ideals of an $R$-module $M$.

Definition 2.4. Let $I$ be an ideal of $R$. We set

$$
\operatorname{Ass}^{\operatorname{comp}}(R / I)=\cup_{j \geq o(I)} \operatorname{Ass}\left(R / I_{\langle j\rangle}\right) .
$$

Lemma 2.5. Let $I$ be an ideal of $R$ with generators in degrees $d_{1}, \ldots, d_{p}, d_{1}<$ $\cdots<d_{p}$. We have

$$
\operatorname{Ass}^{\operatorname{comp}}(R / I)=\operatorname{Ass}\left(R / I_{\left\langle d_{1}\right\rangle}\right) \cup \cdots \cup \operatorname{Ass}\left(R / I_{\left\langle d_{p}\right\rangle}\right) \cup\{\mathbf{m}\} .
$$

In particular, $\operatorname{Ass}^{\mathrm{comp}}(R / I)$ is finite.

Proof. The assertion follows immediately by observing that if $I$ has no generators in degree $j+1$, then $I_{\langle j+1\rangle}=I_{\langle j\rangle} \cap \mathbf{m}^{j+1}$.

Definition 2.6. Let $I$ be an ideal. We denote by $U(I)$ the (finite) union of the prime ideals in $\operatorname{Ass}^{\mathrm{comp}}(R / I) \backslash\{\mathbf{m}\}$.

Proposition 2.7. Let $I$ be an ideal with generators in degrees $d_{1}, \ldots, d_{p}$ with $d_{1}<$ $\cdots<d_{p}$ and set $d_{p+1}=\infty$. The following conditions are equivalent:

(1) $I$ is contracted from $R[\mathbf{m} / \ell]$ for some non-zero linear form $\ell$.

(2) $I$ is contracted from $R[\mathbf{m} / \ell]$ for every non-zero linear form $\ell$ with $\ell \notin U(I)$.

(3) $\left(I_{\langle j\rangle}^{\mathrm{sat}}\right)_{j}=I_{j}$ for every $j \in \mathbf{N}$.

(4) $\left(I_{\left\langle d_{k}\right\rangle}^{\mathrm{sat}}\right)_{j}=I_{j}$ for every $j$ with $d_{k} \leq j<d_{k+1}$ and $k=1, \ldots, p$.

Proof. Obviously (2) implies (1). That (1) implies (3) follows from $I_{j}=\left(I_{\langle j\rangle}^{\mathrm{sat}}: \ell^{\infty}\right)_{j}$, which holds by 2.3. and $\left(I_{\langle j\rangle}^{\mathrm{sat}}: \ell^{\infty}\right)_{j} \supseteq\left(I_{\langle j\rangle}^{\mathrm{sat}}\right)_{j} \supseteq I_{j}$. For (3) implies (2) one notes that if $\ell \notin U(I)$, then we have $I_{\langle j\rangle}^{\text {sat }}: \ell^{\infty}=I_{\langle j\rangle}^{\text {sat }}$ and by assumption $\left(I_{\langle j\rangle}^{\text {sat }}\right)_{j}=I_{j}$. It follows then from 2.3 that $I$ is contracted from $R[\mathbf{m} / \ell]$. Finally, that (3) and (4) are equivalent follows from the observation that if $I$ has no generators in degree $j+1$, then $I_{\langle j+1\rangle}=I_{\langle j\rangle} \cap \mathbf{m}^{j+1}$ and hence $I_{\langle j+1\rangle}^{\mathrm{sat}}=I_{\langle j\rangle}^{\mathrm{sat}}$. 
Proposition 2.8. Every componentwise linear ideal of $R$ is contracted.

Proof. Since $I$ is componentwise linear, we have $\operatorname{reg}\left(I_{\langle j\rangle}\right)=j$ for every $j$ and hence $I_{j}=\left(I_{\langle j\rangle}\right)_{j}=\left(I_{\langle j\rangle}^{\text {sat }}\right)_{j}$. The result follows by $2.7(2)$.

In dimension 3 or higher contracted ideals need not be componentwise linear.

Example 2.9. $\left(x_{1}^{2}, x_{2}^{2}\right)$ is contracted but not componentwise linear in $K\left[x_{1}, x_{2}, x_{3}\right]$.

The following definition is due to Rees. We adapt it to the graded case.

Definition 2.10. An ideal $I \subset R$ is said to be $\mathbf{m}$-full if there exists a non-zero linear form $\ell$ in $R$ such that $I \mathbf{m}: \ell=I$.

Ideals which are $\mathbf{m}$-full are studied in $[\mathrm{W1}, \mathrm{W} 2, \mathrm{~W} 3, \mathrm{G}$. It is easy to see that if $I$ is $\mathbf{m}$-full, then $I: \ell=I: \mathbf{m}$. Moreover, if $I$ is $\mathbf{m}$-full then $I \mathbf{m}: \ell=I$ holds for a general linear form $\ell$. By 2.3 we have immediately that:

Proposition 2.11. Every contracted ideal of $R$ is $\mathbf{m}$-full.

The following example shows that the converse of 2.11 does not hold.

Example 2.12. The ideal $I=\left(x_{1}^{3}, x_{2}^{3}, x_{1}^{2} x_{3}\right)+\left(x_{1}, x_{2}, x_{3}\right)^{4}$ of $K\left[x_{1}, x_{2}, x_{3}\right]$ is $\mathbf{m}$ full. But $I$ is not contracted and $I \mathbf{m}$ is not $\mathbf{m}-$ full.

We recall that an element $a$ of $R$ is said to be integral over $I$ if it satisfies an equation of the form $a^{t}+r_{1} a^{t-1}+\cdots+r_{t}=0$, with $r_{i} \in I^{i}$ for every $i=1, \ldots, t$. The elements of $R$ which are integral over $I$ form an ideal, the integral closure of $I$, denoted by $\bar{I}$. An ideal is said to be integrally closed if it coincides with its integral closure.

Proposition 2.13. Let $\ell \in R_{1} \backslash U(I)$ and $S=R[\mathbf{m} / \ell]$. Then

$$
I \subseteq I S \cap R \subseteq \bar{I}
$$

Proof. By 2.3 we have for every $j$

$$
(I S \cap R)_{j}=\left(I_{\langle j\rangle}^{\mathrm{sat}}\right)_{j} .
$$

Hence for every $f \in(I S \cap R)_{j}$ we have $f \mathbf{m}^{k} \subseteq I_{\langle j\rangle} \mathbf{m}^{k}$ for some $k$. The "determinant trick" implies that $f \in \overline{I_{\langle j\rangle}}$. In particular, $f \in \bar{I}$.

As a corollary we have:

Corollary 2.14. Every integrally closed ideal of $R$ is contracted.

Under the assumption that $I$ is $\mathbf{m}$-primary 2.14 is proved in [DC1, Lemma 3.3]. Further in [G, 2.4] it is proved that integrally closed ideals are $\mathbf{m}$-full in a much more general context. Summing up, we have seen that the following implications hold:

$$
\begin{gathered}
\text { Componentwise linear } \Longrightarrow \text { Contracted } \Longrightarrow \mathbf{m}-\text { full } \\
\Uparrow{ }^{\Uparrow} \\
\text { Integrally closed }
\end{gathered}
$$

In dimension 2, componentwise linear, contracted and $\mathbf{m}$-full are equivalent properties, but, as seen in 2.9 and 2.12 in dimension 3 and higher they differ.

For an $R$-module $M$ we denote by length $(M)$ its length. 
Lemma 2.15. Let $I$ be an $\mathbf{m}$-primary, $\mathbf{m}$-full ideal of order $d$. For every ideal $J$ containing $I$ and for every $\ell$ such that $I \mathbf{m}: \ell=I$ one has

$$
\mu(I)-\mu(J)=\operatorname{length}(\mathbf{m} J / \mathbf{m} I+\ell J) .
$$

It follows that $\mu(I) \geq \mu(J)$ and, in particular, $\mu(I) \geq \mu\left(\mathbf{m}^{d}\right)$.

Proof. See [G, Lemma 2.2. (2)].

One says that $I$ has the Rees property if $\mu(I) \geq \mu(J)$ for every ideal $J \supseteq I$. Under the assumption that $I$ is componentwise linear ideal, the inequality $\mu(I) \geq \mu\left(\mathbf{m}^{d}\right)$ is proved in $[\mathrm{CHH}, 3.4]$. A sort of Rees property is still valid for $\mathbf{m}$-full ideals not necessarily $\mathbf{m}$-primary. We refer to [CHH, 3.2] for the corresponding result for componentwise linear ideals.

Proposition 2.16. Let $I$ and $J$ be ideals of $R$. Assume that $I$ is $\mathbf{m}$-full, $I \subseteq J$ and $I_{t}=J_{t}$ for $t \gg 0$. Then $\mu(I) \geq \mu(J)$.

Proof. First we remark that if $I$ is $\mathbf{m}$-full, then $I+\mathbf{m}^{t}$ is $\mathbf{m}$-full for every integer $t>0$. Now, since $I+\mathbf{m}^{t} \subseteq J+\mathbf{m}^{t}$ and $I+\mathbf{m}^{t}$ is $\mathbf{m}$-primary and $\mathbf{m}$-full ideal, it follows that $\mu\left(I+\mathbf{m}^{t}\right) \geq \mu\left(J+\mathbf{m}^{t}\right)$ by 2.15. Since $I_{t}=J_{t}$ for $t \gg 0$, the inequality $\mu\left(I+\mathbf{m}^{t}\right) \geq \mu\left(J+\mathbf{m}^{t}\right)$ for $t \gg 0$ implies that $\mu(I) \geq \mu(J)$.

Proposition 2.17. Let $I \subset R$ be an ideal of order $d$ and let $\ell$ be a non-zero linear form. Assume that $I+(\ell)=\mathbf{m}^{d}+(\ell)$. Then

(1) if $I$ is $\mathbf{m}$-primary, then $\mu(I) \leq \mu\left(\mathbf{m}^{d}\right)$.

(2) $I=I_{\langle d\rangle}+\ell(I: \ell)$.

(3) $\operatorname{dim} R / I_{\langle d\rangle} \leq 1$.

Proof. (1) If $I+(\ell)=\mathbf{m}^{d}+(\ell)$ holds for a linear form, then it holds for a generic linear form. Thus we may consider a sequence $y_{1}, \ldots, y_{n}$ of generic linear forms in $R$ with $I+\left(y_{1}\right)=\mathbf{m}^{d}+\left(y_{1}\right)$, and set

$$
\alpha_{i}(I)=\operatorname{length}\left(\left[I+\left(y_{1}, \ldots, y_{i}\right)\right]: y_{i+1} /\left[I+\left(y_{1}, \ldots, y_{i}\right)\right]\right) .
$$

By [CHH, 1.2], we have $\mu(I) \leq \sum_{i=0}^{n-1} \alpha_{i}(I)$. We remark that $\alpha_{0}(I)=\operatorname{length}(I$ : $\left.y_{1} / I\right)$. By the exact sequence:

$$
0 \rightarrow \frac{I: y_{1}}{I} \rightarrow \frac{R}{I} \rightarrow \frac{R}{I} \rightarrow \frac{R}{I+\left(y_{1}\right)} \rightarrow 0
$$

it follows that length $\left(I: y_{1} / I\right)=\operatorname{length}\left(R /\left(I+\left(y_{1}\right)\right)\right)$. Since $I+\left(y_{1}\right)=\mathbf{m}^{d}+\left(y_{1}\right)$, we have $\alpha_{0}(I)=\operatorname{length}\left(R / \mathbf{m}^{d}+\left(y_{1}\right)\right)$. Moreover for every integer $i \geq 1$ we have $\left[\left(y_{1}, \ldots, y_{i}\right)+I\right]: y_{i+1} /\left[\left(y_{1}, \ldots, y_{i}\right)+I\right]=\left[\left(y_{1}, \ldots, y_{i}\right)+\mathbf{m}^{d}\right]: y_{i+1} /\left[\left(y_{1}, \ldots, y_{i}\right)+\right.$ $\left.\mathbf{m}^{d}\right]$. Then $\alpha_{i}(I)=\alpha_{i}\left(\mathbf{m}^{d}\right)$ and the result follows since $\sum_{i=0}^{n-1} \alpha_{i}(I)=\sum_{i=0}^{n-1} \alpha_{i}\left(\mathbf{m}^{d}\right)$ and $\sum_{i=0}^{n-1} \alpha_{i}\left(\mathbf{m}^{d}\right)=\mu\left(\mathbf{m}^{d}\right)$.

(2) The inclusion $\supseteq$ is obvious. To prove the other inclusion we note that by assumption $\mathbf{m}^{d} \subseteq I_{\langle d\rangle}+(\ell)$. Thus $I \subseteq \mathbf{m}^{d}+(\ell) \subseteq I_{\langle d\rangle}+(\ell)$, in particular $I \subseteq$ $I_{\langle d\rangle}+(\ell) \cap I=I_{\langle d\rangle}+\ell(I: \ell)$.

(3) By assumption, $\mathbf{m}^{d} \subseteq I_{\langle d\rangle}+(\ell)$, that is $\mathbf{m}=\sqrt{\ell} \bmod I_{\langle d\rangle}$. The conclusion follows by Krull hauptidealsatz.

We are ready to prove the following theorem.

Theorem 2.18. Let $I$ be an $\mathbf{m}$-primary ideal of order $d$ such that $I+(\ell)=\mathbf{m}^{d}+(\ell)$ for some non-zero linear form $\ell$. The following conditions are equivalent: 
(1) $\mu(I)=\mu\left(\mathbf{m}^{d}\right)$,

(2) I is $\mathbf{m}$-full,

(3) I is contracted,

(4) I is componentwise linear.

Proof. The implications $(4) \Longrightarrow(3) \Longrightarrow(2)$ hold in general by 2.8, 2.11, That (2) implies (1) follows by 2.17(1) and 2.15 It remains to prove (1) implies (4). We may assume that $I+(\ell)=\mathbf{m}^{d}+(\ell)$ for a general linear form. With the notation of the proof of 2.17, one sees that the assumption (1) can be stated as $\mu(I)=\sum_{i=0}^{n-1} \alpha_{i}(I)$. Then by $[\mathrm{CHH}, 2.3,1.5]$, we conclude that $I$ is componentwise linear.

In dimension 2 products of contracted ideals are contracted. This is not true in higher dimension.

Example 2.19. Let $R=K\left[x_{1}, x_{2}, x_{3}\right]$, and $I=\left(x_{1}^{2}, x_{1} x_{2}^{2}, x_{2}^{2} x_{3}^{2}\right)$. The ideal $I$ is componentwise linear and hence contracted and $\mathbf{m}$-full. But $I^{2}$ is not $\mathbf{m}$-full (therefore not contracted and not componentwise linear). Take $J=I+\mathbf{m}^{5}$ to get an $\mathbf{m}$-primary example.

The following result will be useful in the next section.

Theorem 2.20. Let $I, J$ be componentwise linear ideals. Let $d$ be the order of $I$ and assume that $\operatorname{dim} R / I_{\langle d\rangle} \leq 1$. Then $I J$ is componentwise linear.

Proof. First assume that $I$ is generated in degree $d$. One has $(I J)_{d+s}=I_{d} J_{s}$ for every $s \in \mathbf{N}$. Now since $\operatorname{dim} R / I_{\langle d\rangle} \leq 1$, by [CH, 2.5], $\operatorname{reg}\left(I_{\langle d\rangle} J_{\langle s\rangle}\right)=d+s$. Hence $I J$ is componentwise linear.

Assume now that $I$ has generators in various degrees. Let $y_{1}, \ldots, y_{n}$ be a generic sequence of linear forms. For $1 \leq p \leq n$ denote by $H_{1}\left(y_{1}, \ldots, y_{p}, R / I J\right)$ the first homology of the Koszul complex of $R / I J$ with respect to $y_{1}, \ldots, y_{p}$. In order to prove that $I J$ is componentwise linear, by $\mathrm{CHH}, 1.5,2.2]$, it suffices to prove that $\mathbf{m} H_{1}\left(y_{1}, \ldots, y_{p}, R / I J\right)=0$ for every $p$. Since $\operatorname{dim} R / I_{\langle d\rangle} \leq 1$ and $\operatorname{reg}\left(I_{\langle d\rangle}+\left(y_{1}\right)\right) \leq$ $\operatorname{reg}\left(I_{\langle d\rangle}\right)=d$ we deduce that $I+\left(y_{1}\right)=\mathbf{m}^{d}+\left(y_{1}\right)$. Consider the Koszul complex:

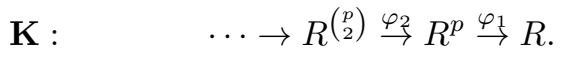

We have to prove that $\mathbf{m}\left(\alpha_{1}, \ldots, \alpha_{p}\right) \in \operatorname{Image}\left(\varphi_{2}\right)+I J R^{p}$ for every $\left(\alpha_{1}, \ldots, \alpha_{p}\right) \in$ $R^{p}$ satisfying $\varphi_{1}\left(\alpha_{1}, \ldots, \alpha_{p}\right) \in I J$.

Since $I+\left(y_{1}\right)=\mathbf{m}^{d}+\left(y_{1}\right)$, then by $2.17(2)$, we have $I=I_{\langle d\rangle}+y_{1}\left(I: y_{1}\right)$. Thus

$$
I J=\left[I_{\langle d\rangle}+y_{1}\left(I: y_{1}\right)\right] J=I_{\langle d\rangle} J+y_{1}\left(I: y_{1}\right) J .
$$

As consequence we may write $\alpha_{1} y_{1}+\alpha_{2} y_{2}+\cdots+\alpha_{p} y_{p}=a+b y_{1}$ with $a \in I_{\langle d\rangle} J$ and $b \in\left(I: y_{1}\right) J$, that is $\left(\alpha_{1}-b\right) y_{1}+\alpha_{2} y_{2}+\cdots+\alpha_{p} y_{p} \in I_{\langle d\rangle} J$ which is componentwise linear by the first part of the proof, thus $\mathbf{m}\left(\alpha_{1}-b, \alpha_{2} \ldots, \alpha_{p}\right) \in \operatorname{Image}\left(\varphi_{2}\right)+$ $I_{\langle d\rangle} J R^{p} \subseteq$ Image $\left(\varphi_{2}\right)+I J R^{p}$. The conclusion follows by noting that $\mathbf{m} b \in J \mathbf{m}(I$ : $\left.y_{1}\right)=J \mathbf{m}(I: \mathbf{m}) \subseteq J I$.

\section{The Classes $\mathcal{C}$ and $\mathcal{C}^{*}$}

In this section we define and study the properties of a class of $\mathbf{m}$-primary ideals of $R=K\left[x_{1}, \ldots, x_{n}\right]$ denoted by $\mathcal{C}$ and of its subclass $\mathcal{C}^{*}$. Before giving the formal definition let us recall few notions that are needed in the sequel. Given an ideal $I$ with $\operatorname{dim} R / I=t$, the multiplicity $e(R / I)$ of $R / I$ is, by definition, $(t-1)$ ! times 
the leading coefficient of the Hilbert polynomial of $R / I$ if $t>0$ and it is $\operatorname{dim}_{K} R / I$ otherwise. In particular, by definition, we have $e(R / R)=0$.

Definition 3.1. We define $\mathcal{C}$ to be the class of the ideals $I$ of $R$ of finite colength such that:

(1) $I+(\ell)=\mathbf{m}^{o(I)}+(\ell)$ for some non-zero linear form $\ell$,

(2) $I$ verifies one of the equivalent conditions of 2.18

We also set

$$
\mathcal{C}^{*}=\{I \in \mathcal{C}: I \text { is integrally closed }\} .
$$

Remark 3.2. (1) In the definition above we say "finite colength" and not simply "m-primary" because we want $\mathcal{C}$ to contain $R$.

(2) If $n=2$, then $\mathcal{C}$ is the class of contracted ideals.

(3) It follows from $[\mathrm{CHH}, 3.4]$ that $\mathcal{C}$ can be also defined as the class of finite colength ideals $I$ which are componentwise linear with $\mu(I)=\mu\left(\mathbf{m}^{o(I)}\right)$.

The next example shows that $\mathcal{C}$ cannot be defined as the class of "contracted ideals with $\mu(I)=\mu\left(\mathbf{m}^{o(I)}\right)$ ".

Example 3.3. In $K\left[x_{1}, x_{2}, x_{3}\right]$ the ideal $I=\left(x_{1}^{2}, x_{2} x_{3}\right)+\mathbf{m}^{3}$ is integrally closed, hence contracted and $\mathbf{m}$-full. Furthermore $\mu(I)=\mu\left(\mathbf{m}^{2}\right)$. But $I \notin \mathcal{C}$.

However we have:

Lemma 3.4. Let $I$ be an ideal of $R$ of finite colength and order $d$. If both $I$ and $\mathbf{m} I$ are $\mathbf{m}$-full and $\mu(I)=\mu\left(\mathbf{m}^{d}\right)$, then $\mathbf{m} I \in \mathcal{C}$.

Proof. Since $I$ is $\mathbf{m}$-full and $\mu(I)=\mu\left(\mathbf{m}^{d}\right)$, then by 2.15 applied with $J=\mathbf{m}^{d}$ we deduce that there exists $\ell$ such that $\mathbf{m}^{d+1}+(\ell)=I \mathbf{m}+(\ell)$. Since $\mathbf{m} I$ is $\mathbf{m}$-full, we conclude that $\mathbf{m} I \in \mathcal{C}$.

The class $\mathcal{C}$ is closed under the product and the integral closure.

Proposition 3.5. If $I, J \in \mathcal{C}$, then $I J \in C$ and $\bar{I} \in \mathcal{C}^{*}$.

Proof. Set $d=o(I)$ and $d_{1}=o(J)$. Choose $\ell$ such that $I+(\ell)=\mathbf{m}^{d}+(\ell)$ and $J+(\ell)=\mathbf{m}^{d_{1}}+(\ell)$. Hence $\mathbf{m}^{d+d_{1}}+(\ell) \subseteq I J+(\ell)$. Since the opposite inclusion is obvious, one has $\mathbf{m}^{d+d_{1}}+(\ell)=I J+(\ell)$. Furthermore by 2.17 the dimension of $R / I_{\langle d\rangle}$ is $\leq 1$. Hence $I J$ is componentwise linear by 2.20 . Hence $I J \in \mathcal{C}$. As for $\bar{I}$ one notes that, by degree reasons, $o(\bar{I})=d$ and $\mathbf{m}^{d} \subseteq I+(\ell) \subseteq \bar{I}+(\ell)$. Being integrally closed, $\bar{I}$ is contracted. It follows that $\bar{I} \in \mathcal{C}^{*}$.

Example 2.12 shows that the class defined by the conditions " $m$-full and $\mu(I)=$ $\mu\left(\mathbf{m}^{o(I)}\right)$ ", which properly contains $\mathcal{C}$, is not closed under the product.

In dimension 2, to every contracted ideal $I$ of order $d$ one associates its characteristic form $g(I)$ which is, by definition, the GCD of the elements in $I_{d}$. Zariski proved [ZS, Appendix 5] a factorization property for contracted ideals in dimension 2. The factors are characterized by having pairwise coprime characteristic forms which are powers of irreducible forms. Now we want to generalize Zariski's theorem to the class $\mathcal{C}$. To this end we will give another description of the ideals in it.

Definition 3.6. We denote by $\mathcal{A}$ the set of the families $\mathcal{Q}=\left\{Q_{j}\right\}_{j \in \mathbf{N}}$ of homogeneous ideals of $R$ satisfying the following conditions:

(1) $Q_{j} \subseteq Q_{j+1}$ for every $j$, 
(2) $Q_{j}=R$ for $j \gg 0$,

(3) whenever $Q_{j} \neq R$, the ideal $Q_{j}$ is saturated and $\operatorname{dim} R / Q_{j}=1$.

Given $\mathcal{Q}=\left\{Q_{i}\right\} \in \mathcal{A}$, let $d_{0}=\operatorname{reg}\left(Q_{0}\right)$. For every $k \in \mathbf{N}$ we set

$$
I(\mathcal{Q}, k)=\oplus_{j \in \mathbf{N}}\left(Q_{j}\right)_{d_{0}+k+j} .
$$

We have:

Proposition 3.7. For every $\mathcal{Q}=\left\{Q_{j}\right\} \in \mathcal{A}$ and for every $k \in \mathbf{N}$, one has

$$
I(\mathcal{Q}, k) \in \mathcal{C} \text {. }
$$

Proof. Since $Q_{j} \subseteq Q_{j+1}$ we have $R_{1} Q_{j} \subseteq Q_{j+1}$ and hence $R_{1}\left(Q_{j}\right)_{d_{0}+j+k} \subseteq$ $\left(Q_{j+1}\right)_{d_{0}+j+1+k}$. This proves that $I(\mathcal{Q}, k)$ is an ideal. If $Q_{0}=R$, then $I(\mathcal{Q}, k)=\mathbf{m}^{k}$ for all $k \geq 0$. Assume now that $Q_{0} \neq R$. Let $\ell$ be a linear form non-zero-divisor on $R / Q_{0}$. Since $\operatorname{reg} Q_{0}=d_{0}$, the ideal $Q_{0}+(\ell)$ is 0 -dimensional of regularity $d_{0}$. It follows that $\mathbf{m}^{d_{0}} \subseteq Q_{0}+(\ell)$. Therefore $\mathbf{m}^{d_{0}+k} \subseteq\left(Q_{0}\right)_{\left\langle d_{0}+k\right\rangle}+(\ell) \subseteq I(\mathcal{Q}, k)+(\ell)$ and hence $\mathbf{m}^{d_{0}+k}+(\ell)=I(\mathcal{Q}, k)+(\ell)$. It remains to prove that $I(\mathcal{Q}, k)$ is componentwise linear, that is, $\left(Q_{j}\right)_{\left\langle d_{0}+h\right\rangle}$ has a linear resolution for every $h \in \mathbf{N}$. By assumption $Q_{j} \subseteq Q_{j+1}$ and they define Cohen-Macaulay rings of the same dimension or are equal to $R$. It follows that $\operatorname{reg}\left(Q_{j}\right) \geq \operatorname{reg}\left(Q_{j+1}\right)$. Hence $\operatorname{reg}\left(Q_{j}\right) \leq \operatorname{reg}\left(Q_{0}\right)=d_{0}$ for every $j$. Then for every $h \geq 0$ we have $\left(Q_{j}\right)_{\left\langle d_{0}+h\right\rangle}$ has a linear resolution. This proves the assertion.

Given an ideal $I$ in $\mathcal{C}$ of order $d$, for every $j \geq 0$, we set

$$
Q_{j}(I)=\left(I_{\langle d+j\rangle}\right)^{\mathrm{sat}} \text {. }
$$

Proposition 3.8. Let $I \in \mathcal{C}$ and $d=o(I)$. For every $j \in \mathbf{N}$ set $\mathcal{Q}(I)=\left\{Q_{j}(I)\right\}$ and $d_{0}=\operatorname{reg}\left(Q_{0}(I)\right)$. Then $\mathcal{Q}(I) \in \mathcal{A}$ and $d \geq d_{0}$.

Proof. Since $I_{\langle d\rangle}$ has dimension $\leq 1$, then $Q_{j}(I)$ is saturated of dimension 1 or it is equal to $R$. Moreover $I_{\langle d+j\rangle} R_{1} \subseteq I_{\langle d+j+1\rangle} \subseteq I_{\langle d+j+1\rangle}^{\text {sat }}$. Hence $Q_{j}(I) \subseteq Q_{j+1}(I)$. We have $d_{0}=\operatorname{reg}\left(Q_{0}(I)\right) \leq \operatorname{reg} I_{\langle d\rangle}=d$.

As a consequence we have:

Theorem 3.9. With the notation of 3.7 and 3.8 the applications

$$
\varphi: \mathcal{A} \times \mathbf{N} \longrightarrow \mathcal{C} \text { and } \psi: \mathcal{C} \rightarrow \mathcal{A} \times \mathbf{N}
$$

defined by $\varphi(\mathcal{Q}, k)=I(\mathcal{Q}, k)$ and $\psi(I)=\left(\mathcal{Q}(I), d-d_{0}\right)$ are inverse to each other.

Proof. That the maps are well-defined follows from 3.7 and 3.8. That are inverse to each other is a straightforward verification based on the observation that if $J$ is a saturated ideal generated in degree $\leq t$, then $J_{\langle t\rangle}=J \cap \mathbf{m}^{t}$ and hence $J_{\langle t\rangle}^{\text {sat }}=J$.

We need to recall now few facts about the ideal transform. Let $S=R[\mathbf{m} / \ell]$ where $\ell$ is a non-zero linear form. Clearly $\mathbf{m} S=(\ell) S$ and for every homogeneous element $f$ of degree $d$ one has $f=\left(f / \ell^{d}\right) \ell^{d}$ in $S$. Hence for every ideal $I$ of order $d$ we have

$$
I S=\ell^{d} I^{\prime},
$$

where $I^{\prime}$ is an ideal of $S$. The ideal $I^{\prime}$ is called the ideal transform of $I$ in $S$.

Proposition 3.10. Let $I, J \in \mathcal{C}$ with $o(I) \geq o(J)$. The following facts are equivalent: 
(1) $Q_{j}(I)=Q_{j}(J)$ for every $j$.

(2) $I \mathbf{m}^{s}=J \mathbf{m}^{r}$ for some $r, s \in \mathbf{N}$.

(3) $I=J \mathbf{m}^{r}$ where $r=o(I)-o(J)$.

(4) $I^{\prime}=J^{\prime}$ in $S=R[\mathbf{m} / \ell]$ for every linear form $\ell$.

(5) $I^{\prime}=J^{\prime}$ in $S=R[\mathbf{m} / \ell]$ for a linear form $\ell$ not in $U(I) \cup U(J)$.

Proof. Conditions (1), (2) and (3) are equivalent by 3.7 3.8 and 3.9, That (3) implies (4) is clear by construction. That (4) implies (5) is obvious. Assume (5) and set $r=o(I)-o(J)$. Then $I S=\ell^{o(I)} I^{\prime}$ and $J \mathbf{m}^{r} S=\ell^{r} \ell^{o(J)} J^{\prime}=I S$. Since $J \in \mathcal{C}$, we have $J \mathbf{m}^{r} \in \mathcal{C}$ by 3.5. Hence $I$ and $J \mathbf{m}^{r}$ are contracted from $S$. Since they have the same extension, it follows that $I=J \mathbf{m}^{r}$.

Definition 3.11. For $I, J \in \mathcal{C}$ we set $I \equiv J$ if $I$ and $J$ verify the equivalent conditions of 3.10 .

In a different setting a similar equivalent relation is introduced in $[\mathrm{L}$.

The extension $R \rightarrow R\left[\mathbf{m} / x_{n}\right]$ can be identified with the $K$-algebra homomorphism $\phi: R \rightarrow R$ sending $x_{i} \rightarrow x_{i} x_{n}$ for $i=1, \ldots, n-1$ and $x_{n}$ to $x_{n}$. One has $\phi\left(f\left(x_{1}, \ldots, x_{n}\right)\right)=x_{n}^{d} f\left(x_{1}, \ldots, x_{n-1}, 1\right)$ for every form of degree $d$. Denote by $\phi^{\prime}: R \rightarrow K\left[x_{1}, \ldots, x_{n-1}\right]$ the dehomogenization map, that is, the $K$-algebra homomorphism sending $x_{i} \rightarrow x_{i}$ for $i=1, \ldots, n-1$ and $x_{n}$ to 1 . So we have $\phi(f)=x_{n}^{d} \phi^{\prime}(f)$ for every form of degree $d$.

Let $I \in \mathcal{C}$ of order $d$. Let $P_{1}, \ldots, P_{m}$ be the minimal primes of $Q_{0}(I)=I_{\langle d\rangle}^{\text {sat }}$, necessarily homogeneous of dimension 1 (with $m=0$ if $Q_{0}(I)=R$, that is, $I=\mathbf{m}^{d}$ ). Note that, by construction, $I$ is contracted from any extension $R[\mathbf{m} / \ell]$ with $\ell \notin \cup P_{i}$. After a change of coordinates, we may assume that $x_{n} \notin \cup_{i=1}^{m} P_{i}$ and take $\ell=x_{n}$. We may write $I=\sum_{j \geq 0} I_{\langle j+d\rangle}$ and so

$$
\phi(I) R=\sum_{j \geq 0} \phi\left(I_{\langle j+d\rangle}\right) R=x_{n}^{d} \sum_{j \geq 0} \phi^{\prime}\left(I_{\langle j+d\rangle}\right) x_{n}^{j} .
$$

It follows that

$$
I^{\prime}=\sum_{j \geq 0} \phi^{\prime}\left(I_{\langle j+d\rangle}\right) x_{n}^{j}
$$

that is

$$
I^{\prime}=\left\{\sum_{j} a_{j} x_{n}^{j}: a_{j} \in \phi^{\prime}\left(I_{\langle j+d\rangle}\right)\right\} .
$$

Proposition 3.12. With the notation above, we have:

$$
\sqrt{I^{\prime}}=\cap_{i=1}^{m}\left(\phi^{\prime}\left(P_{i}\right) R+\left(x_{n}\right)\right)
$$

and $\phi^{\prime}\left(P_{i}\right) R+\left(x_{n}\right)$ are distinct maximal ideals of $R$.

Proof. By definition, $Q_{j}(I)=I_{\langle j+d\rangle}^{\text {sat }}$. Hence for some $u \in \mathbf{N}$ one has $x_{n}^{u} Q_{j}(I) \subseteq$ $I_{\langle j+d\rangle} \subseteq Q_{j}(I)$ which implies

$$
\phi^{\prime}\left(I_{\langle j+d\rangle}\right)=\phi^{\prime}\left(Q_{j}(I)\right) .
$$

It follows that

$$
I^{\prime}=\sum_{j \geq 0} \phi^{\prime}\left(Q_{j}(I)\right) x_{n}^{j}
$$


Since $Q_{j}(I)=R$ for $j \gg 0$ we have that $x_{n}^{j} \in I^{\prime}$ for $j \gg 0$. As a consequence we have:

$$
\sqrt{I^{\prime}}=\sqrt{\phi^{\prime}\left(Q_{0}(I)\right) R+\left(x_{n}\right)}=\sqrt{\phi^{\prime}\left(Q_{0}(I)\right)} R+\left(x_{n}\right)
$$

The known properties of the dehomogenization, see for instance [KR, Section 4.3], guarantee that $\sqrt{\phi^{\prime}\left(Q_{0}(I)\right)}=\cap_{i=1}^{m} \phi^{\prime}\left(P_{i}\right)$. The rest follows since $\phi^{\prime}\left(P_{i}\right)$, as an ideal of $K\left[x_{1}, \ldots, x_{n-1}\right]$, is maximal and $\phi^{\prime}\left(P_{i}\right) \neq \phi^{\prime}\left(P_{j}\right)$ for $i \neq j$.

The next result generalizes Zariski's factorization theorem for contracted ideals [ZS, Appendix 5, Thm. 1] to the class $\mathcal{C}$. The role played in [ZS by the characteristic form is played here by the ideal $Q_{0}(I)$. We call $Q_{0}(I)$ the characteristic ideal of $I$.

Theorem 3.13. Let $I \in \mathcal{C}$ and let $P_{1}, \ldots, P_{m}$ be the minimal prime ideals of $Q_{0}(I)$. We have:

(1) There exist $L_{1}, \ldots, L_{m} \in \mathcal{C}$ such that

$$
I \equiv L_{1} L_{2} \cdots L_{m}
$$

and every $L_{i}$ has a $P_{i}$-primary characteristic ideal.

(2) The $L_{i}$ 's satisfying (1) are uniquely determined by I up to $\equiv$. In particular, $Q_{j}\left(L_{i}\right)=Q_{j}(I) R_{P_{i}} \cap R$.

Proof. First we prove that the $L_{i}$ 's defined as in (2) satisfy (1) and then we prove the uniqueness of the $L_{i}$. For $i=1, \ldots, m$ and $j \in \mathbf{N}$ set $Q_{i}=\left\{Q_{j}(I) R_{P_{i}} \cap R\right\}_{j \in \mathbf{N}}$. Then set $L_{i}=I\left(Q_{i}, 0\right)$. By construction, $L_{i} \in \mathcal{C}$ and $Q_{j}\left(L_{i}\right)=Q_{j}(I) R_{P_{i}} \cap R$ and hence $Q_{0}\left(L_{i}\right)$ is $P_{i}$-primary. By 3.5 we have $L_{1} L_{2} \cdots L_{m} \in \mathcal{C}$. According to 3.10 , to prove (1) it is enough to show that

$$
I^{\prime}=L_{1}^{\prime} L_{2}^{\prime} \cdots L_{m}^{\prime}
$$

in $S=R[\mathbf{m} / \ell]$ for a general linear form $\ell$. After a change of coordinates, we may assume that $x_{n} \notin P_{i}$ for every $i$ and hence take $\ell=x_{n}$. Using formula (3.1) to describe $I^{\prime}$ and the $L_{i}^{\prime}$ 's, (3.2) becomes equivalent to

$$
\phi^{\prime}\left(Q_{j}(I)\right)=\sum_{*} \prod_{k=1}^{m} \phi^{\prime}\left(Q_{j_{k}}\left(L_{k}\right)\right)
$$

for all $j$, where the sum $\sum_{*}$ of the right hand side is extended to all the $j_{1}, \ldots, j_{m}$ such that $j_{1}+j_{2}+\cdots+j_{m}=j$. Equivalently,

$$
\phi^{\prime}\left(Q_{j}(I)\right)=\phi^{\prime}\left(\sum_{*} \prod_{k=1}^{m} Q_{j_{k}}\left(L_{k}\right)\right) .
$$

If we show that:

Claim 3.14. $Q_{j}(I)$ is the saturation of $\sum_{*} \prod_{k=1}^{m} Q_{j_{k}}\left(L_{k}\right)$

then we are done because two homogeneous ideals with the same saturation become equal after dehomogenization. To prove the claim we localize $\sum_{*} \prod_{k=1}^{m} Q_{j_{k}}\left(L_{k}\right)$ at each $P_{i}$. What we get is $\left(\sum Q_{j_{i}}\left(L_{i}\right)\right) R_{P_{i}}$ where the sum is exteded to $j_{i} \leq j$, that is, $Q_{j}\left(L_{i}\right) R_{P_{i}}$. Since $Q_{j}\left(L_{i}\right)=Q_{j}(I) R_{P_{i}} \cap R$ we have $Q_{j}\left(L_{i}\right) R_{P_{i}}=Q_{j}(I) R_{P_{i}}$. This proves the claim. Now assume that there are other ideals $W_{i} \in C$ such that $I \equiv W_{1} \cdots W_{m}$ and $Q_{0}\left(W_{i}\right)$ is $P_{i}$-primary. Then $I^{\prime}=W_{1}^{\prime} \cdots W_{m}^{\prime}$. Since by Proposition 3.12 the $W_{i}^{\prime}$ are primary to distinct maximal ideals, we have that $I^{\prime}=W_{1}^{\prime} \cap \cdots \cap W_{m}$ is a primary decomposition. By the uniqueness of minimal 
components in primary decompositions, we have $W_{i}^{\prime}=L_{i}^{\prime}$ and hence $W_{i} \equiv L_{i}$ as desired.

We present now a formula for the Hilbert series of $I$ in terms of the Hilbert series of the ideals $L_{1}, \ldots, L_{m}$ appearing in the factorization of Theorem 3.13. If $\operatorname{dim} R=2$, this has been already done in [CDJR, 3.10].

Since $I$ is an $\mathbf{m}$-primary ideal, then length $\left(I^{k} / I^{k+1}\right)$ is finite for every integer $k$. The Hilbert function $\operatorname{HF}_{I}(k)$ of $I$ is defined as

$$
\operatorname{HF}_{I}(k)=\operatorname{length}\left(I^{k} / I^{k+1}\right) \text {. }
$$

The Hilbert series of $I$ is

$$
\operatorname{HS}_{I}(z)=\sum_{k \geq 0} \operatorname{HF}_{I}(k) z^{k}
$$

It is well known that the Hilbert series is of the form

$$
\operatorname{HS}_{I}(z)=\frac{h_{0}(I)+h_{1}(I) z+\ldots+h_{s}(I) z^{s}}{(1-z)^{n}}
$$

with $h_{i}(I) \in \mathbf{Z}$ for every $i, h_{0}(I)=\operatorname{length}(R / I)$ and $e(I)=\sum_{i=0}^{s} h_{i}(I)$ is the multiplicity of $I$. By definition, the h-polynomial of $I$ is

$$
h_{I}(z)=h_{0}(I)+h_{1}(I) z+\ldots+h_{s}(I) z^{s} .
$$

Lemma 3.15. Let $I$ be in $\mathcal{C}$ and let $I \equiv L_{1} L_{2} \cdots L_{m}$ be the factorization of 3.13. One has

$$
\operatorname{length}\left(\mathbf{m}^{d} / I\right)=\sum_{i=1}^{m} \operatorname{length}\left(\mathbf{m}^{d_{i}} / L_{i}\right)
$$

where $d=o(I)$ and $d_{i}=o\left(L_{i}\right)$ for every $i=1, \ldots, m$.

Proof. Since reg $Q_{j}(I) \leq d$, then $\operatorname{dim}_{K}\left(R_{d+j} / I_{d+j}\right)$ coincides with the multiplicity of $R / Q_{j}(I)$. Hence

$$
\operatorname{length}(R / I)=\operatorname{length}\left(R / \mathbf{m}^{d}\right)+\sum_{i \geq 0} e\left(R / Q_{j}(I)\right) .
$$

Thus length $\left(\mathbf{m}^{d} / I\right)=\sum_{j \geq 0} e\left(R / Q_{j}(I)\right)$. Since we know that $Q_{j}(I)=Q_{j}\left(L_{1}\right) \cap$ $\cdots \cap Q_{j}\left(L_{m}\right)$, the multiplicity formula $[\mathrm{BH}, 4.7 .8]$ implies that $e\left(R / Q_{j}(I)\right)=$ $\sum_{i=1}^{m} e\left(R / Q_{j}\left(L_{i}\right)\right)$ and thus

$$
\begin{aligned}
\operatorname{length}\left(\mathbf{m}^{d} / I\right) & =\sum_{j \geq 0} e\left(R / Q_{j}(I)\right)=\sum_{j \geq 0} \sum_{i=1}^{m} e\left(R / Q_{j}\left(L_{i}\right)\right) \\
& =\sum_{i=1}^{m} \sum_{j \geq 0} e\left(R / Q_{j}\left(L_{i}\right)\right)=\sum_{r=1}^{m} \operatorname{length}\left(\mathbf{m}^{d_{r}} / L_{r}\right) .
\end{aligned}
$$

Proposition 3.16. With the notations of 3.15 we have:

$$
\mathrm{HS}_{I}(z)=\sum_{j=1}^{m} \operatorname{HS}_{L_{j}}(z)+\mathrm{HS}_{\mathbf{m}^{d}}(z)-\sum_{j=1}^{m} \mathrm{HS}_{\mathbf{m}^{d_{j}}}(z)
$$


and in particular

$$
e(I)=\sum_{j=1}^{m} e\left(L_{j}\right)+d^{n}-\sum_{j=1}^{m} d_{j}^{n} .
$$

Proof. Note that for every integer $k$ the factorization of $I^{k}$ is:

$$
I^{k} \equiv L_{1}^{k} L_{2}^{k} \cdots L_{m}^{k}
$$

and hence

$$
\operatorname{length}\left(\mathbf{m}^{k d} / I^{k}\right)=\sum_{i=1}^{m} \operatorname{length}\left(\mathbf{m}^{k d_{i}} / L_{i}^{k}\right) .
$$

To conclude, first rewrite length $\left(\mathbf{m}^{k d} / I^{k}\right)$ as length $\left(R / I^{k}\right)-\operatorname{length}\left(R / \mathbf{m}^{k d}\right)$ and similarly for the $L_{i}$ 's and then sum up.

Example 3.17. In $K[x, y, z]$ consider the ideal $I=\left(x^{3}, y^{3}, z^{3}, x y, y z, x z\right)$ of $\mathcal{C}$. We have $Q_{0}(I)=(x y, y z, x z)$ and $Q_{j}(I)=R$ for $j>0$. It follows from 3.13 that $I \equiv L_{1} L_{2} L_{3}$ where $L_{1}=\left(x^{2}, y, z\right), L_{2}=\left(x, y^{2}, z\right), L_{3}=\left(x, y, z^{2}\right)$. To get an equality of ideals, we have to multiply the left hand side by $(x, y, z)$ :

$$
(x, y, z)\left(x^{3}, y^{3}, z^{3}, x y, y z, x z\right)=\left(x^{2}, y, z\right)\left(x, y^{2}, z\right)\left(x, y, z^{2}\right) .
$$

Taking into account that $d=2, d_{1}=d_{2}=d_{3}=1$ and that the $L_{i}$ 's are complete intersections, we may apply 3.16 and get:

$$
\mathrm{HS}_{I}(z)=3 \frac{2}{(1-z)^{3}}+\frac{4+4 z}{(1-z)^{3}}-3 \frac{1}{(1-z)^{3}}
$$

that is

$$
\operatorname{HS}_{I}(z)=\frac{7+4 z}{(1-z)^{3}}
$$

The ideal of Example 3.17 appears in $[\mathrm{C}]$ and $[\mathrm{L}]$.

Theorem 3.18. Let $I \in \mathcal{C}$. Then

(1) $\overline{\mathbf{m} I}=\mathbf{m} \bar{I}$.

(2) $I \in \mathcal{C}^{*}$ if and only if $\mathbf{m} I \in \mathcal{C}^{*}$.

Proof. (1) The inclusion $\mathbf{m} \bar{I} \subseteq \overline{\mathbf{m} I}$ holds in general, see [HS, 1.1.3]. Using the characterization of integral closure by means of valuations, one shows that

$$
\overline{\mathbf{m} I}: \ell=\bar{I}
$$

for every ideal $I$ and general linear form $\ell$, see the proof of [ $\mathrm{H} 2,3.1,3.3]$ for details. Since $I \in \mathcal{C}$, then $\mathbf{m} I+(\ell)=\mathbf{m}^{o(I)+1}+(\ell)$ is integrally closed. Then $\overline{\mathbf{m} I} \subseteq$ $\overline{\mathbf{m} I+(\ell)}=\mathbf{m} I+(\ell)$. Hence

$$
\overline{\mathbf{m} I}=(\mathbf{m} I+(\ell)) \cap \overline{\mathbf{m} I}=\mathbf{m} I+\ell(\overline{\mathbf{m} I}: \ell)=\mathbf{m} I+\ell \bar{I} \subseteq \mathbf{m} \bar{I} .
$$

(2) If $I \in \mathcal{C}^{*}$ then (1) implies $\mathbf{m} I \in \mathcal{C}^{*}$. Conversely if $\mathbf{m} I \in \mathcal{C}^{*}$ then $\overline{\mathbf{m} I}: \ell=$ $\mathbf{m} I: \ell=\bar{I}$. Since $I$ is $\mathbf{m}$-full, it follows $I=\bar{I}$.

Special cases of Theorem 3.18 and Proposition 3.19]appear in DC1. In general, even for a normal ideal $I$ the product $\mathbf{m} I$ need not be integrally closed, see [DC2, Example 7.1].

Proposition 3.19. We have:

(1) If $I \in \mathcal{C}^{*}$ then $I^{\prime}$ is integrally closed. 
(2) If $I^{\prime}$ is integrally closed and $I$ is contracted, then $I$ is integrally closed. In particular if $I \in \mathcal{C}$, then $I \in \mathcal{C}^{*}$ if and only if $I^{\prime}$ is integrally closed.

Proof. Since $I S=\ell^{d} I^{\prime}$ and $S$ is a polynomial ring (hence normal), then (1) follows if we prove that $I S$ is integrally closed. Consider the integral equation

$$
s^{m}+a_{1} s^{m-1}+\cdots+a_{m}=0
$$

with $s \in S, a_{i} \in(I S)^{i}$. For every $i=0, \ldots, m$, we may write $a_{i}=b_{i} / \ell^{\alpha}$ with $b_{i} \in I^{i} \mathbf{m}^{\alpha}$ and $\alpha$ a fixed positive integer. Multiplying by $\ell^{m \alpha}$ we get an equation among elements of $R$, namely

$$
t^{m}+b_{1} t^{m-1}+\cdots+\left(b_{2} \ell^{\alpha}\right) t^{m-2}+\cdots+\left(b_{m} / \ell^{\alpha}\right)=0
$$

where $t=s \ell^{\alpha}$ and $b_{i} \ell^{(i-1) \alpha} \in I^{i} \mathbf{m}^{i \alpha}$. Since $I \mathbf{m}^{\alpha}$ is integrally closed by 3.18, it follows that $t=s \ell^{\alpha} \in I \mathbf{m}^{\alpha}$. Hence $s \in I S$.

We prove now (2). Let $x \in R$ and $a_{i} \in I^{i}$ such that

$$
x^{m}+a_{1} x^{m-1}+\cdots+a_{m}=0
$$

and we claim that $x \in I$. Note that $a_{i} / \ell^{i d} \in\left(I^{\prime}\right)^{i}$ and

$$
\left(x / \ell^{d}\right)^{m}+a_{1} / \ell^{d}\left(x / \ell^{d}\right)^{m-1}+\cdots+a_{m} / \ell^{d m}=0 .
$$

Since $I^{\prime}$ is integrally closed, it follows that $x / \ell^{d} \in I^{\prime}$, that is, $x \in I S$. Since $I$ is contracted we have $x \in I$.

Theorem 3.20. Given $I \in \mathcal{C}$ let $I \equiv L_{1} L_{2} \cdots L_{m}$ be the factorization of [3.13, Then $I \in \mathcal{C}^{*}$ if and only if $L_{j} \in \mathcal{C}^{*}$ for every $j=1, \ldots, m$.

Proof. Assume that $I$ is integrally closed. By $3.19(1), I^{\prime}=L_{1}^{\prime} \cdots L_{m}^{\prime}$ is integrally closed. Since $L_{1}^{\prime}, \ldots, L_{m}^{\prime}$ are primary to distinct maximal ideals, by localizing and contracting back one has that each $L_{i}^{\prime}$ is integrally closed. Hence each $L_{i}$ is integrally closed by 3.19 (2). Conversely if $L_{i}$ is integrally closed for every $i=1, \ldots, m$, then $L_{i}^{\prime}$ is integrally closed by $3.19(1)$. It follows that so is $I^{\prime}=L_{1}^{\prime} \cdots L_{m}^{\prime}$ since $L_{1}^{\prime} \cdots L_{m}^{\prime}=L_{1}^{\prime} \cap \cdots \cap L_{m}^{\prime}$. Finally by 3.19 (2) we conclude that $I$ is integrally closed.

The following examples show that the class $\mathcal{C}^{*}$ is not closed under product (for $n \geq 3$ ) and powers (for $n \geq 4$ ):

Example 3.21. (1) The ideals $(x, y)^{3}+\left(x^{2} z\right)+\mathbf{m}^{4}$ and $(x, y)^{3}+\left(y^{2} z\right)+\mathbf{m}^{4}$ of $K[x, y, z]$ are in $\mathcal{C}^{*}$ but not their product.

(2) The ideal $\left(x^{2}, y^{3}, z^{7}, x y^{2}, x y z^{2}, x z^{4}, y z^{5}, y^{2} z^{3}, y z^{5}\right) \cap \mathbf{m}^{7}+\mathbf{m}^{8}$ of $K[x, y, z, t]$ is in $\mathcal{C}^{*}$ but not its square.

Nevertheless, as an immediate consequence of Theorem 3.20, we have:

Corollary 3.22. Let $I, J \in \mathcal{C}^{*}$ such that $Q_{0}(I)+Q_{0}(J)$ is $\mathbf{m}$-primary. Then $I J \in \mathcal{C}^{*}$.

Another corollary is:

Corollary 3.23. With the notation of 3.13 we have

$$
\bar{I} \equiv \overline{L_{1}} \overline{L_{2}} \cdots \overline{L_{m}}
$$

and $Q_{0}\left(\overline{L_{i}}\right)$ is $P_{i}$-primary. 
Proof. Combining [HS, Exercise 1.1, p. 20] with 3.18, we get:

$$
\bar{I} \equiv \overline{\overline{L_{1}}} \overline{L_{2}} \cdots \overline{L_{m}} .
$$

The conclusion follows from 3.20 provided we prove that $Q_{0}\left(\overline{L_{i}}\right)$ is $P_{i}$-primary. So assume that $L \in \mathcal{C}$ has order $d$ and $Q_{0}(L)$ is $P$-primary for some 1-dimensional prime $P$. Set $J=\bar{L}$. By degree reasons, $J_{d} \subset \overline{L_{\langle d\rangle}}$ and $\overline{L_{\langle d\rangle}} \subseteq \sqrt{L_{\langle d\rangle}}=P$. Hence $J_{\langle d\rangle} \subseteq P$ which implies that $Q_{0}(J)$ is $P$-primary.

\section{The Goto-classes $\mathcal{G}$ and $\mathcal{G}^{*}$}

Consider the following subclass of $\mathcal{C}$ :

Definition 4.1. We define the Goto-class $\mathcal{G}$ to be the set of the ideals $I \in \mathcal{C}$ such that:

(1) The minimal primes $P_{1}, \ldots, P_{m}$ of $Q_{0}(I)$ are geometrically prime, equivalently, each $P_{i}$ is generated by $n-1$ linearly independent linear forms in $R=K\left[x_{1}, \ldots, x_{n}\right]$ (e.g. $K$ is algebraically closed).

(2) For every $j \in \mathbf{N}$ the primary components of $Q_{j}(I)$ are powers of the $P_{i}$ 's. That is,

$$
Q_{j}(I)=\cap_{i=1}^{m} P_{i}^{\alpha_{i j}}
$$

with $\alpha_{i j} \in \mathbf{N}$.

Further we set:

$$
\mathcal{G}^{*}=\{I \in \mathcal{G}: I \text { is integrally closed }\} .
$$

In dimension two $\mathcal{G}=\mathcal{C}$ and it coincides with the whole class of contracted ideals. Our goal is to show that the Goto-classes $\mathcal{G}$ and $\mathcal{G}^{*}$ behave, to a certain extent and respectively, as the class of contracted ideals and the class of integrally closed ideals in dimension 2. The factorization in Theorem 3.13 will allow to reduce most of the problems to the case of ideals in $\mathcal{G}$ with a primary characteristic ideal. So we will discuss in some details the properties of these ideals.

Let $P$ be a geometrically prime ideal of $R$ of dimension 1 . Let $L \in \mathcal{G}$ of order $d$ such that $Q_{0}(L)$ is $P$-primary. Then $Q_{j}(L)=P^{\alpha_{j}}$ where the $\alpha_{j}$ 's form a weakly decreasing integral sequence with $\alpha_{j}=0$ for $j \gg 0$. Hence $L$ is described by the triplet $P,\left\{\alpha_{j}\right\}$ and $d$. We give another description of $L$ that best suits our needs. Indeed, one shows that there exists a uniquely determined sequence of integers $0=a_{0}<a_{1}<\cdots<a_{d}$ such that

$$
L=\sum_{i=0}^{d} P^{d-i} \ell^{a_{i}}
$$

where $\ell$ is any linear form not in $P$. To emphasize the dependence of $L$ on $P$ and the sequence $a_{0}, \ldots, a_{d}$ we will denote $L$ by $L(P, a)$, that is,

$$
L(P, a)=\sum_{i=0}^{d} P^{d-i} \ell^{a_{i}}
$$

Example 4.2. Let $R=K\left[x_{1}, x_{2}, x_{3}\right]$ and $P=\left(x_{1}, x_{2}\right)$. Associated with the sequence $\alpha=(5,3,3,2,0,0 \ldots)$ and with $d=6$ we have the ideal $L$ whose components are $L_{6+j}=\left(P^{\alpha_{j}}\right)_{6+j}$ for $j \geq 0$. We can write $L$ as $L(P, a)=\sum P^{d-i} x_{3}^{a_{i}}$ where $a=(0,1,3,4,7,9,10)$. 
Given two sequences of integers $a=\left(a_{0}, \ldots, a_{d}\right)$ and $b=\left(b_{0}, \ldots, b_{e}\right)$ we define their product $a b$ to be the sequence $\left(c_{0}, \ldots, c_{d+e}\right)$ where $c_{j}=\min \left\{a_{r}+b_{s}: r+s=j\right\}$. Furthermore we denote by $a^{(k)}$ the product of $a$ with itself $k$ times. By the very definition one has:

$$
L(P, a) L(P, b)=L(P, a b) \text { and } L(P, a)^{k}=L\left(P, a^{(k)}\right)
$$

for every $a, b$ and $P$. We have:

Proposition 4.3. Let $a=\left(a_{0}, \ldots, a_{d}\right) \in \mathbf{N}^{d+1}$ be an increasing sequence with $a_{0}=0$.

(1) There exists an increasing sequence $a^{\prime}=\left(a_{0}^{\prime}, \ldots, a_{d}^{\prime}\right)$ with $a_{0}^{\prime}=0$ (uniquely determined by $a)$ such that for every $n>1$ and for every 1-dimensional geometrically prime ideal $P$ of $R=K\left[x_{1}, \ldots, x_{n}\right]$ one has $\overline{L(P, a)}=L\left(P, a^{\prime}\right)$.

(2) The following conditions are equivalent:

(i) $L(P, a)$ is integrally closed for every $n>1$ and for every 1-dimensional geometrically prime ideal $P$ of $R$.

(ii) $L(P, a)$ is integrally closed for some $n>1$ and some 1-dimensional geometrically prime ideal $P$ of $R$.

(ii) $a=a^{\prime}$.

Proof. (1) Let $n>1$ and let $P$ be a 1-dimensional geometrically prime ideal of $R$. Choosing bases properly, we may assume that $P=\left(x_{1}, \ldots, x_{n-1}\right)$ and $\ell=x_{n}$ so that $L(P, a)$ is a monomial ideal. The integral closure of a monomial ideal $I$ is the ideal generated by the monomials $m$ such that $m^{k} \in I^{k}$ for some $k>$ 0. A monomial $m=m_{1} x_{n}^{d-j}$ with $m_{1}$ supported on $x_{1}, \ldots, x_{n-1}$ satisfies $m^{k}=$ $m_{1}^{k} x_{n}^{k d-k j} \in L(P, a)^{k}$ iff $\operatorname{deg} m_{1}^{k}=k \operatorname{deg} m_{1} \geq\left(a^{(k)}\right)_{k j}$ iff $\operatorname{deg} m_{1} \geq\left(a^{(k)}\right)_{k j} / k$. Hence setting

$$
a_{j}^{\prime}=\min \left\{\left\lceil\left(a^{(k)}\right)_{k j} / k\right\rceil: k>0\right\}
$$

we get (1). Statement (2) follows immediately from (1).

Given a 1-dimensional geometrically prime ideal $P$ of $R$ and numbers $d, t \in \mathbf{N}$ with $d \leq t$ we set

$$
J_{P}(d, t)=\overline{P^{d}+\mathbf{m}^{t}}
$$

equivalently

$$
J_{P}(d, t)=\overline{\left(\ell_{1}^{d}, \ldots, \ell_{n-1}^{d}, \ell^{t}\right)}
$$

where $P=\left(\ell_{1}, \ldots, \ell_{n-1}\right)$ and $\ell$ is a linear form not in $P$. By construction, $J_{P}(d, t) \in$ $\mathcal{G}^{*}$ and its characteristic ideal is $P^{d}$ unless $t=d$. Hence $J_{P}(d, t)$ must be of the form $L(P, a)$ for a sequence $a$. Indeed a simple computation shows that:

$$
J_{P}(d, t)=L(P, a)
$$

where $a=\left(a_{0}, \ldots, a_{d}\right)$ with $a_{i}=\lceil i t / d\rceil$ for $i=0, \ldots, d$.

We say that an ideal $I$ is simple if it cannot be written as a product of proper ideals.

Remark 4.4. It is an easy exercise and part of the folklore of the subject that $\overline{\left(x_{1}^{d}, x_{2}^{t}\right)}$ is simple in $K\left[x_{1}, x_{2}\right]$ iff $\operatorname{GCD}(d, t)=1$ and that every simple integrally closed ideal of $K\left[x_{1}, x_{2}\right]$ with characteristic form equal to $x_{1}$ is of the form $\overline{\left(x_{1}^{d}, x_{2}^{t}\right)}$.

Proposition 4.5. Let $a=\left(a_{0}, \ldots, a_{d}\right) \in \mathbf{N}^{d+1}$ be an increasing sequence with $a_{0}=0$ and $P$ a 1-dimensional geometrically prime ideal of $R$. Then the following conditions are equivalent: 
(1) $L(P, a)$ is integrally closed, simple and different from $\mathbf{m}$.

(2) there exists $t>d$ such that $\operatorname{GCD}(d, t)=1$ and $L(P, a)=J_{P}(d, t)$.

(3) there exists $t>d$ such that $\operatorname{GCD}(d, t)=1$ and $a_{i}=\lceil i t / d\rceil$ for $i=0, \ldots, d$.

Proof. The result follows from 4.34 .4 and the following claim:

Claim 4.6. $L(P, a)$ is integrally closed and simple in $R=K\left[x_{1}, \ldots, x_{n}\right]$ if and only if $L\left(\left(x_{1}\right), a\right)$ is integrally closed and simple in $K\left[x_{1}, x_{2}\right]$.

To prove the claim assume first that $L(P, a)$ is integrally closed and simple. Then $L\left(\left(x_{1}\right), a\right)$ is integrally closed by 4.3 . If, by contradiction, $L\left(\left(x_{1}\right), a\right)$ is not simple, then $L\left(\left(x_{1}\right), a\right)=I J$ with $I, J$ integrally closed. Hence $I$ and $J$ are of the form $I=L\left(\left(x_{1}\right), b\right)$ and $J=L\left(\left(x_{1}\right), c\right)$. It follows that $L(P, a)=L(P, b) L(P, c)$ contradicting the fact that $L(P, a)$ is simple.

Viceversa, assume that $L\left(\left(x_{1}\right), a\right)$ is integrally closed and simple. Then $L(P, a)$ is integrally closed by 4.3 If, by contradiction, $L(P, a)$ is not simple, then $L(P, a)=$ $I J$ with $I, J$ proper ideals. Since $\mathbf{m}^{u} \subset L(P, a) \subseteq I$ it follows that $\sqrt{I}=\mathbf{m}$ and for the same reason $\sqrt{J}=\mathbf{m}$. After a change of coordinates, we may assume that $P=\left(x_{1}, \ldots, x_{n-1}\right)$ and consider the $K$-algebra homomorphism $\psi: R \rightarrow K\left[x_{1}, x_{2}\right]$ sending $x_{i}$ to $x_{1}$ for $i<n$ and $x_{n}$ to $x_{2}$. We have $L\left(\left(x_{1}\right), a\right)=\psi(L(P, a))=$ $\psi(I) \psi(J)$ and $\psi(I)$ and $\psi(J)$ are proper since $\psi(I) \subseteq \psi(\mathbf{m})=\left(x_{1}, x_{2}\right)$ and similarly for $\psi(J)$. This contradicts the assumptions and proves the claim.

Next we show that the factorization of Theorem 3.13 restricts to the class $\mathcal{G}$.

Proposition 4.7. We have:

(1) Let $I \in \mathcal{C}$ be such that the minimal primes of $Q_{0}(I)$ are geometrically prime. Let $I \equiv L_{1} \cdots L_{m}$ be the factorization of [3.13, Then $I \in \mathcal{G}$ iff $L_{i} \in \mathcal{G}$ for every $i$.

(2) $\mathcal{G}$ is closed under product.

(3) If $I \in \mathcal{G}$ then $\bar{I} \in \mathcal{G}^{*}$.

Proof. (1) Let $\left\{P_{1}, \ldots, P_{m}\right\}$ the minimal primes of $Q_{0}(I)$. By 3.13 we know that $Q_{j}(I) R_{P_{i}}=Q_{j}\left(L_{i}\right) R_{P_{i}}$ and this implies the assertion.

(2) Let $I, J \in \mathcal{G}$. Set $d=o(I)$ and $c=o(J)$. We have to show that for every $P \in \operatorname{Min}\left(Q_{0}(I J)\right)$ and for every $j$ we have $(I J)_{\langle d+c+j\rangle} R_{P}$ is a power of $P R_{P}$. Note that $(I J)_{\langle d+c+j\rangle}=\sum_{i=0}^{j} I_{\langle d+i\rangle} J_{\langle c+j-i\rangle}$ and that $I_{d+i} R_{P}=P^{a_{i}} R_{P}$ and $J_{\langle c+j-i\rangle} R_{P}=P^{b_{j-i}} R_{P}$ for non-negative integers $a_{i}$ and $b_{i}$. It follows that we have $(I J)_{\langle d+c+j\rangle} R_{P}=P^{t} R_{P}$ where $t=\min \left\{a_{i}+b_{j-i}: i=0, \ldots, j\right\}$.

(3) Let $I \equiv L_{1} \cdots L_{m}$ be the factorization of 3.13 . Then by 3.23 we have $\bar{I} \equiv$ $\prod_{i} \overline{L_{i}}$. By (1) it is enough to show that $\overline{L_{i}} \in \mathcal{G}$. We may hence assume that $I$ is of the form $L(P, a)$. But we have already observed in 4.3 that $\overline{L(P, a)}=L\left(P, a^{\prime}\right)$, which implies that $\overline{L(P, a)} \in \mathcal{G}$.

We can state now the main result of the section.

Theorem 4.8. We have:

(1) $\mathcal{G}^{*}$ is closed under product. In particular, every $I \in \mathcal{G}^{*}$ is normal.

(2) Every $I \in \mathcal{G}^{*}$ has a factorization

$$
I \equiv J_{1} \cdots J_{t}
$$

where $J_{i} \in \mathcal{G}^{*}$ is simple and $Q_{0}\left(J_{i}\right)$ is primary for every $i=1, \ldots, t$. 
(3) In the factorization of (2), the factors $J_{i}$ are uniquely determined by I up to order. Moreover, each $J_{i}$ is of the form $J_{P_{i}}\left(d_{i}, t_{i}\right)$ and $d_{i}<t_{i}$ with $\operatorname{GCD}\left(d_{i}, t_{i}\right)=1$.

Proof. (1) Let $I, J \in \mathcal{G}^{*}$. By 4.7(2) we know that $I J \in \mathcal{G}$ and we have to prove that $I J$ is integrally closed. By 3.13, 4.7(1) and 3.20 we have factorizations $I \equiv L_{1} \cdots L_{m}$ and $J \equiv U_{1} \cdots U_{r}$ and the $L_{i}$ and $U_{j}$ belong to $\mathcal{G}^{*}$. Hence $I J \equiv \prod L_{i} \prod U_{i}$. If $L$ and $U$ have $P$-primary characteristic ideal then the same is true for $L U$. Hence, the factors in the (unique) factorization of 3.13 of $I J$ are of the form $L_{i} U_{j}$ (if $L_{i}$ and $U_{j}$ have $P$-primary characteristic ideal with respect to the same prime) or $L_{i}$ or $U_{j}$. By virtue of 3.20 we may assume right away that $I$ and $J$ have $P$-primary characteristic ideal, say $I=L(P, a)$ and $J=L(P, b)$. Then $I J=L(P, a b)$. Since $I$ and $J$ are integrally closed, the same is true for $L\left(\left(x_{1}\right), a\right)$ and $L\left(\left(x_{1}\right), b\right)$ in $K\left[x_{1}, x_{2}\right]$ by 4.3 . As in dimension 2 the product of integrally closed ideals is integrally closed, we have that $L\left(\left(x_{1}\right), a\right) L\left(\left(x_{1}\right), b\right)=L\left(\left(x_{1}\right), a b\right)$ is integrally closed. By 4.3 it follows that $I J$ is integrally closed.

(2) By virtue of 4.7 we have $I \equiv L_{1} \cdots L_{m}$ with $L_{i} \in \mathcal{G}^{*}$ and $Q_{0}\left(L_{i}\right)$ primary. Hence we may assume that $I=L(P, a)$ for some 1-dimensional geometrically prime ideal $P$ and a sequence $a=\left(a_{0}, \ldots, a_{d}\right)$. By Zarisky factorization theorem [ZS] and 4.4 one has $L\left(\left(x_{1}\right), a\right)=\left(x_{1}, x_{2}\right)^{c} J_{\left(x_{1}\right)}\left(d_{1}, t_{1}\right) \cdots J_{\left(x_{1}\right)}\left(d_{p}, t_{p}\right)$ with $d_{i}<t_{i}$ and $\operatorname{GCD}\left(d_{i}, t_{i}\right)=1$. It follows that $I=\mathbf{m}^{c} J_{P}\left(d_{1}, t_{1}\right) \cdots J_{P}\left(d_{p}, t_{p}\right)$ and hence $I \equiv$ $J_{P}\left(d_{1}, t_{1}\right) \cdots J_{P}\left(d_{p}, t_{p}\right)$. The conclusion follows from 4.5 .

(3) That the factors of the factorization in (2) are of the form $J_{P_{i}}\left(d_{i}, t_{i}\right)$ with $d_{i}<t_{i}$ and $\operatorname{GCD}\left(d_{i}, t_{i}\right)=1$ has been already proved. It remains to prove the uniqueness. Suppose we have two factorizations of $I$ as in (2). By 4.7 and 3.13 we may assume that the characteristic form of $I$ is $P$-primary. Hence we have $I \equiv J_{P}\left(d_{1}, t_{1}\right) \cdots J_{P}\left(d_{p}, t_{p}\right)$ and $I \equiv J_{P}\left(c_{1}, s_{1}\right) \cdots J_{P}\left(c_{q}, s_{q}\right)$ with $d_{i}<t_{i}$ and $\operatorname{GCD}\left(d_{i}, t_{i}\right)=1$ as well as $c_{i}<s_{i}$ and $\operatorname{GCD}\left(c_{i}, s_{i}\right)=1$. As a consequence we have $\mathbf{m}^{a} J_{P}\left(d_{1}, t_{1}\right) \cdots J_{P}\left(d_{p}, t_{p}\right)=\mathbf{m}^{b} J_{P}\left(c_{1}, s_{1}\right) \cdots J_{P}\left(c_{q}, s_{q}\right)$, and it follows that $\left(x_{1}, x_{2}\right)^{a} J_{\left(x_{1}\right)}\left(d_{1}, t_{1}\right) \cdots J_{\left(x_{1}\right)}\left(d_{p}, t_{p}\right)=\left(x_{1}, x_{2}\right)^{b} J_{\left(x_{1}\right)}\left(c_{1}, s_{1}\right) \cdots J_{\left(x_{1}\right)}\left(c_{q}, s_{q}\right)$ in $K\left[x_{1}, x_{2}\right]$. By the uniqueness of the factorization of integrally closed ideals in $K\left[x_{1}, x_{2}\right]$, we have that $p=q$ and, up to the order, $\left(d_{i}, t_{i}\right)=\left(c_{i}, s_{i}\right)$ for $i=1, \ldots, p$. Hence $J_{P}\left(d_{i}, t_{i}\right)=J_{P}\left(c_{i}, s_{i}\right)$ for $i=1, \ldots, p$ proving the assertion.

Remark 4.9. Let I be an $\mathbf{m}$-primary complete intersection ideal of $R$. Goto proved in $[\mathrm{G}$ ] that the following conditions are equivalent:

(1) I is integrally closed.

(2) $I$ is normal.

(3) $I=\left(\ell_{1}, \ldots, \ell_{n-1}, \ell_{n}^{t}\right)$ for linearly independent linear forms $\ell_{1}, \ldots, \ell_{n}$ and some $t>0$.

Complete intersections satisfying these equivalent conditions are called of Goto-type (see [CHKV] $)$. Note that the ideals of Goto-type are in the Goto-class $\mathcal{G}$, they are exactly the ideals of type $J_{P}(1, t)$ used above.

Hence, as a consequence of Theorem 4.8 , we have:

Corollary 4.10. The product of complete intersections of Goto-type is a normal ideal.

In dimension 2, every integrally closed ideal has a Cohen-Macaulay associated graded ring (see [H2], [LT]). This is no longer true in higher dimension and not even 
for normal ideals. The first examples of normal ideals with non Cohen-Macaulay associated graded ring is given by a construction of Cutkosky [C]. Later on Huckaba and Huneke $\mathrm{HuHu}$, Theorem 3.12]) proved that

$$
I=\left(x^{4}\right)+(x, y, z)\left(y^{3}+z^{3}\right)+(x, y, z)^{5} \subseteq K[x, y, z]
$$

is normal, but $\operatorname{gr}_{I^{n}}(R)$ is not Cohen-Macaulay for every $n$.

One might, however, ask:

Question 4.11. Let $I \in \mathcal{G}^{*}$. Is $\operatorname{gr}_{I}(R)$ Cohen-Macaulay?

We show that Question 4.11 has a positive answer in two cases. The first is the following.

Corollary 4.12. Let $I \in \mathcal{G}^{*}$. Then $\operatorname{Rees}(I)$ is normal. In particular, $\operatorname{Rees}(I)$, equivalently $\operatorname{gr}_{I}(R)$, is Cohen-Macaulay if $I$ is monomial in some system of coordinates (e.g. the characteristic ideal of I has at most 2 minimal primes).

Proof. The first assertion follows from 4.8(1). The second follows from the fact that if the characteristic ideal of $I$ has at most 2 minimal primes, then up to a choice of coordinates, we may assume that $I$ is monomial. For a monomial ideal $I$, the normality of Rees $(I)$ implies its Cohen-Macaulayness as proved by Hochster BH, $6.3 .5]$.

To show that 4.11 has a positive answer if $\operatorname{dim} R \leq 3$ we need the following result.

Lemma 4.13. Let $I$ be an $\mathbf{m}$-primary ideal of $R=K\left[x_{1}, \ldots, x_{n}\right]$. If $\operatorname{gr}_{I}(R)$ is Cohen-Macaulay, then the degree of its $h$-polynomial is $\leq n-1$.

Proof. Since the ideal $I$ is $\mathbf{m}$-primary, then $\operatorname{gr}_{I}(R)$ is Cohen-Macaulay if and only if $\operatorname{gr}_{I_{\mathbf{m}}}\left(R_{\mathbf{m}}\right)$ is Cohen-Macaulay; moreover $\operatorname{gr}_{I}(R)$ and $\operatorname{gr}_{I_{\mathbf{m}}}\left(R_{\mathbf{m}}\right)$ have the same Hilbert series. Hence we may reduce the problem to the local case (see for example Remark 2.2. CDJR ). Note that if $J$ is a minimal reduction of $I$, then the $h$ polynomial $h_{I}(z)=h_{0}(I)+h_{1}(I) z+\ldots+h_{s}(I) z^{s}$ coincides with the Hilbert series of the ideal $I / J$. Now by a consequence of Briancon-Skoda HS, 11.1.9], we have $I^{n} \subseteq J$, hence $\operatorname{HF}_{I / J}(n)=\operatorname{length}\left(I^{n}+J / I^{n+1}+J\right)=h_{n}(I)=0$ and the result follows.

Theorem 4.14. Assume that $\operatorname{dim} R \leq 3$. If $I \in \mathcal{G}^{*}$, then $\operatorname{gr}_{I}(R)$ is CohenMacaulay.

Proof. Consider the factorization $I \equiv L_{1} \cdots L_{m}$ of 3.13 , We know by 3.20 and 4.7 that $L_{i} \in \mathcal{G}^{*}$. By 3.16 one has $h_{I}(z)=\sum_{j=1}^{m} h_{L_{j}}(z)+h_{\mathbf{m}^{d}}(z)-\sum_{j=1}^{m} h_{\mathbf{m}^{d_{j}}}(z)$. By 4.12 we know that $\operatorname{gr}_{L_{i}}(R)$ is Cohen-Macaulay for every $i=1, \ldots, m$. That $\operatorname{gr}_{\mathbf{m}^{u}}(R)$ is Cohen-Macaulay for every $u$ is well-know. Thus by 4.13 the degree of $h_{L_{i}}(z) \leq 2$ for every $i=1, \ldots, m$ and the same is true for $h_{\mathbf{m}^{u}}(z)$. It follows that the degree of $h_{I}(z)$ is $\leq 2$. Localizing at $\mathbf{m}$ we may assume that $R$ is local. Let $J$ be a minimal reduction of $I$; since $I$ is integrally closed, by [H1] we have $I^{2} \cap J=J I$. Then the result follows by [GR, 2.2].

\section{REFERENCES}

[BH] Bruns, W., Herzog, J.: Cohen-Macaulay rings, Cambridge Studies in Advanced Mathematics, 39. Cambridge University Press, Cambridge (1993) 
[CH] Conca, A., Herzog, J.: Castelnuovo-Mumford regularity of products of ideals, Collect. Math. $\mathbf{5 4}(2), 137-152(2003)$

[CDJR] Conca, A., De Negri, E., Jayanthan, A.V., M.E.Rossi, M.E.: Graded rings associated with contracted ideals, J. Algebra 284(2), 593-626 (2005)

[CHH] Conca, A., Herzog, J., Hibi, T.: Rigid resolutions and big Betti numbers, Comment. Math. Helv. 79(4), 826-839 (2004)

[CGPU] Corso, A., Ghezzi, L., Polini, C., Ulrich, B.: Cohen-Macaulayness of special fiber rings, Special issue in honor of S.Kleiman, Comm. Algebra 31(8), 3713-3734 (2003)

[CHKV] Corso, A., Huneke, C., Katz, D., Vasconcelos, W.: Integral closure of ideals and annihilators of homology, Commutative algebra, 33-48, Lect. Notes Pure Appl. Math., 244, Chapman Hall/CRC, Boca Raton, FL (2006)

[C] Cutkosky, S.D.: Factorization of complete ideals, J. of Algebra 115(1), 144-149 (1989)

[C1] Cutkosky, S.D.: On unique and almost unique factorization of complete ideals, Amer. J. Math. 111(3), 417-433 (1989)

[C2] Cutkosky, S.D.: On unique and almost unique factorization of complete ideals. II, Invent. Math. 98(1) 59-74 (1989)

[C3] Cutkosky, S.D.: A new characterization of rational surface singularities, Invent. math. 102, 157-177 (1990)

[DC1] D'Cruz, C.: Quadratic transform of complete ideals in regular local rings, Comm. in Algebra, 28 (2), 693-698, (2000)

[DC2] D'Cruz, C.: Integral closedness of $M I$ and the formula of Hoskin Deligne for finitely supported complete ideals, J. of Algebra 304(2), 613-632 (2006)

[D] Deligne, P.: Intersections sur les surfaces regulieres, SGA 7, II, Lecture Notes in Math. no. 340, Springer-Verlag, Berlin-Heidelberg-New York, 1-38 (1973)

[E] Eisenbud, D.: Commutative algebra. With a view toward algebraic geometry, Graduate Texts in Mathematics, 150. Springer-Verlag, New York (1995)

[EM] Eisenbud, D., Mazur, B.: Evolutions, symbolic squares, and Fitting ideals, J. Reine Angew. Math. 488, 189-201 (1997)

[Ga] Gately, J.: Unique factorization of $*$-products of one-fibered monomial ideals, Comm. Algebra 28(7), 3137-3153, (2000)

[Ga1] Gately, J.: *-simple complete monomial ideals, Comm. Algebra 33(8) 2833-2849, (2005)

[G] Goto, S.: Integral closedness of complete intersection ideals, J. Algebra 108, 151-160 (1987)

[GR] Guerrieri, A., Rossi, M.E.: Hilbert coefficients of Hilbert filtrations, J. Algebra 199, 40-61 (1998)

[HHi] Herzog, J., Hibi, T.: Componentwise linear ideals, Nagoya Math. J. 153, 141-153 (1999)

[HH] Hübl, R., Huneke, C.: Fiber cone and the integral closure of ideals, Collect. Math. 52(1), $85-100(2001)$

[HuHu] Huckaba, S., Huneke, C.: Normal ideals in regular rings, J. Reine Angew. Math. 510, 63-82 (1999)

[H] Huneke, C.: The primary components of an integral closure of ideals in 3-dimensional regular local rings, Math. Ann. 275, 617-635 (1986)

[H1] Huneke, C.: Hilbert function and symbolic powers, Michigan J. Math 34, 293-318, (1987)

[H2] Huneke, C.: Complete ideals in two-dimensional regular local rings, Commutative algebra (Berkeley, CA, 1987), 325-338, Math. Sci. Res. Inst. Publ., 15, Springer, New York 1989

[HS] Huneke, C., Swanson, I.: Integral Closure of Ideals, Rings and Modules, London Mathematical Society Lecture Note Series, 336. Cambridge University Press, Cambridge (2006)

[KR] Kreuzer, M., Robbiano, L.: Computational commutative algebra 2, Springer-Verlag, Berlin (2005)

[L] Lipman, J.: On Complete Ideals in Regular Local Rings, Algebraic Geometry and Commutative Algebra (in honor of M. Nagata), 203-231 (1987)

[LT] Lipman, J., Teissier, B.: Pseudorational local rings and a theorem of Briançon-Skoda about integral closure of ideals, Mich. Math. J. 28, 97-116 (1981)

[W1] Watanabe, J.: m-full ideals, Nagoya Math. J., 106, 101-111 (1987)

[W2] Watanabe, J.: The syzygies of $\mathbf{m}$-full ideals, Math.Proc.Camb.Phil.Soc. 109, 7-13 (1991)

[W3] Watanabe, J.: m-full ideals II, Math.Proc.Camb.Phil.Soc. 111, 231-240 (1992)

[Z] Zariski, O.: Polynomial ideals defined by infinitely near base points, Amer. J. Math. 60, 151-204 (1938) 
[ZS] Zariski, O., Samuel, P.: Commutative Algebra, Vol.2, The University Series in Higher Mathematics. D. Van Nostrand Co., Inc., Princeton, N. J.-Toronto-London-New York 1960

Dipartimento di Matematica, Universitá di Genova, Via Dodecaneso 35, I-16146 GenOVA, ITALY

E-mail address: conca@dima.unige.it, denegri@dima.unige.it, rossim@dima.unige.it 